\title{
Impact of direct carotid artery perfusion in acute type $A$ aortic dissection involving the common carotid artery
}

\author{
Kayo Sugiyama ${ }^{1} \cdot$ Hirotaka Watanuki $^{1} \cdot$ Yasuhiro Futamura ${ }^{1} \cdot$ Masaho Okada $^{1} \cdot$ Tatsunori Oiwa $^{1} \cdot$ Satoshi Makino $^{1}$. \\ Katsuhiko Matsuyama ${ }^{1}$
}

Received: 5 December 2020 / Accepted: 25 March 2021 / Published online: 2 April 2021

(C) The Japanese Association for Thoracic Surgery 2021

\begin{abstract}
Objectives Acute type A aortic dissection complicated with brain ischemia is associated with significantly higher mortality risks. Even if rescued with central aortic repair, some patients develop permanent postoperative neurological deficiency postoperatively. We recently introduced direct common carotid artery perfusion for acute type A aortic dissection involving the common carotid artery. This study introduced this technique to prevent postoperative neurological deficiency by comparing brain protection strategies.

Methods Among 91 acute type A aortic dissection patients treated at our hospital during August 2015-October 2020, the common carotid artery was involved in $19(21 \%)$, which had $>90 \%$ stenosis in either of the carotid arteries on preoperative contrast-enhanced computed tomography. Twelve patients underwent conventional selective cerebral perfusion during August 2015-December 2018 and seven patients underwent direct carotid artery perfusion during January 2019-October 2020. We assessed patient characteristics, surgical courses, clinical outcomes, and neurological outcomes.

Results The mean age was 69 (range 39-84) years; 17 patients were male (89\%). Eight patients (42\%) had neurological symptoms. Concomitant procedures, postoperative neurological symptoms, and late mortality were significantly associated with conventional selective cerebral perfusion. Five selective cerebral perfusion patients experienced worsened neurological symptoms and two died of broad cerebral ischemia. No direct carotid artery perfusion patient died during hospitalization or experienced worsened neurological outcomes.

Conclusions Direct carotid artery perfusion may be useful in aortic dissection with brain ischemia because it does not aggravate neurological symptoms and is not associated with intraoperative cerebral infarction. However, it may be ineffective when cerebral infarction has already developed.
\end{abstract}

Keywords Cerebral malperfusion · Acute aortic dissection · Direct carotid artery perfusion

\section{Introduction}

Brain malperfusion caused by acute type A aortic dissection (AAAD) represents a major risk factor for early death or disability, although early surgical results for AAAD have recently improved $[1,2]$. Previous reports have proposed that central repair of entry closure and true lumen reinstallation should be performed first to improve early survival rate and neurological outcomes; however, some patients have

Kayo Sugiyama

kayotaro3@gmail.com

1 Department of Cardiac Surgery, Aichi Medical University Hospital, 1-1 Yazakokarimata, Nagakute, Aichi 480-1195, Japan developed irreversible neurological deficits after central aortic repair [3, 4]. Moreover, it is difficult to determine which patients with neurological symptoms will recover after surgery $[5,6]$. Therefore, Gomibuchi et al. introduced concept of "imaging cerebral malperfusion" for subclinical patients, which is defined as having occlusion or narrowing of common carotid artery in a preoperative computed tomography regardless of symptoms [6].

The pathophysiology of brain complications in acutely dissected patients is largely due to a compressed true lumen by the false lumen, static or dynamic [7]. It is often multifactorial, frequently coexisting with circulatory collapse, sequelae of acute hypoxia, and thromboembolism originating from the false lumen [7]. However, as a main cause of postoperative neurological deficiency, the possibility of 
thromboembolism owing to thrombi derived from a thrombosed false lumen has been noted [6,8-10]. According to these reports, the removal of thrombi from the false lumen before the initiation of selective cerebral perfusion (SCP) is extremely important.

To prevent irreversible brain injury, preoperative assessment and intraoperative management are important; however, the optimal intraoperative strategy remains controversial [11]. Axillary artery perfusion might be effective in limited cases but may not restore the true lumen and may cause systemic hypoperfusion [12,13]. Direct ascending aortic cannulation was established as a promising new method; however, it is suboptimal for brain protection [14]. Direct perfusion of the common carotid artery is a reasonable technique to resolve preoperative cerebral malperfusion $[7,15,16]$. However, their main concept differs from ous in some respects. Therefore, this study introduced the technique of direct carotid artery perfusion (DCP) and aimed to prevent postoperative neurological deficiency by comparing brain protection strategies.

\section{Methods}

\section{Study design and population}

This single-center retrospective cohort study reviewed records of consecutive patients who underwent emergency surgical repair for AAAD at Aichi Medical University Hospital from August 2015 to October 2020. Data from the institutional database were retrospectively selected from cases of aortic disease with the involvement of the common carotid artery, which was defined as having over $90 \%$ stenosis in either of the common carotid arteries compressed by a dilated false lumen as observed on preoperative contrast-enhanced computed tomography (CT). According to the change in our strategy, patients were devided into two groups; patients who underwent conventional SCP between August 2015 and December 2018 and those who underwent DCP between January 2019 and October 2020. All procedures were performed according to the tenets of the Helsinki Declaration, and the Ethics Committee of Aichi Medical University Hospital approved the study on July 31, 2020 (Approval Number, 2020-077). All patients provided written consent for their clinical data to be used for scientific presentations or publications.

Diagnosis of AAAD was confirmed using contrastenhanced CT in our hospital or other previous hospitals. Involvement of the common carotid artery was defined as having over $90 \%$ stenosis on contrast-enhanced CT in either of the common carotid arteries resulting from compression by a dilated false lumen regardless of neurological symptoms. In our institute, cerebral protection was considered for all patients with an involvement of the common carotid artery because of the following reasons. First, it is unclear which cases will develop cerebral infarction as some asymptomatic patients could develop irreversible neurological deficits after central aortic repair. Second, there is often no sufficient time to assess which cases will develop cerebral infarction after surgery. Carotid ultrasonography was conducted in some patients to further examine cerebral perfusion. Preoperative symptoms related to neurological deficiency were defined as experiencing coma, hemiplegia, hemiparesis, or a transient ischemic attack. Patients with disorders of consciousness owing to hemodynamic collapse arising from cardiac tamponade, severe aortic valve regurgitation, coronary malperfusion, and aortic rupture were excluded. Furthermore, patients with a history of cerebrovascular disease, defined as a history of intracranial bleeding or infarction with permanent neurologic dysfunction, were excluded. In our institute, preoperatively comatose patients were excluded from undergoing open surgical repair. When the patients recovered from coma, open surgery was considered.

Data on patient demographics (age and sex) and comorbidities (hypertension, smoking history, chronic respiratory disease, chronic renal disease, history of coronary artery disease, and family history related to aortic dissection) were recorded. Hypertension was defined as a casual blood pressure of 149/90 $\mathrm{mmHg}$ and/or ongoing antihypertensive treatment. Chronic respiratory disease was defined as chronic obstructive lung disease or pulmonary fibrosis requiring specific medication. Chronic renal disease was defined as a serum creatinine concentration $\geq 2 \mathrm{mg} / \mathrm{dL}$. Coronary artery disease was defined as a history of coronary revascularization. Specific preoperative complications related to aortic dissection such as other organ malperfusion, aortic valve regurgitation, cardiac tamponade, shock hemodynamics, cardiac arrest and resuscitation were also recorded.

Patient cases were separated into two groups based on the strategy for brain protection, which changed with time: 12 patients underwent conventional SCP and seven underwent DCP. Both early and late clinical outcomes were assessed. Perioperative parameters, including time from onset to start of cerebral perfusion, operation time, cardiopulmonary bypass time, aortic cross clamp time, cerebral perfusion time, circulatory arrest time, and minimal body temperature, were assessed. Values of continuous bilateral cerebral regional oxygen saturation monitored using the INVOS ${ }^{\mathrm{TM}}$ Cerebral/Somatic Oximetry Adult Sensors (Medtronic, Minneapolis, MN) were also recorded and assessed. The German Registry for Acute Aortic Dissection Type A (GERAADA) score is an effective tool to predict the 30-day mortality rate of patients undergoing surgery for AAAD [17, 18]. Thus, the GERAADA score in all patients was calculated and assessed. 
Early outcomes included early mortality, cause of death, neurological outcomes, and other complications. Respiratory complications were defined as long-term ventilator dependence that required tracheostomy. Postoperative symptoms related to neurological deficiency included deep coma, hemiplegia, and hemiparesis. Neurological worsening was defined as progression of neurological deficiency compared to preoperative symptoms. Late outcomes included late mortality, cause of death, major adverse aortic events (MAAE), and neurological events. MAAE comprised a composite of either major aortic events or major aortic re-intervention. Major aortic events included rupture or re-dissection of the aorta. Major aortic re-interventions included additional thoracic endovascular aortic repair or a major surgical graft revision.

\section{Operative procedures}

Central aortic repair was performed for entry exclusion and true lumen reinstallation. The extent of graft replacement was based on the position of the primary entry. When the entry was in the ascending aorta, hemiarch replacement was selected, and when the entry was in the aortic arch, total arch or partial arch replacement was selected. An intra-bladder or pharyngeal temperature of $25{ }^{\circ} \mathrm{C}$ was set as the target.

In cases of conventional $\mathrm{SCP}$, cardiopulmonary bypass was instituted via bicaval drainage and both femoral artery and right axillary artery cannulation. In cases of DCP, the stenosed right or left carotid artery was exposed at the beginning of the surgery. The vessel was then cannulated directly using a 12 Fr Perfusion Cannula Combined Pressure Monitoring ${ }^{\mathrm{TM}}$ (SUMITOMO BAKELITE, Tokyo, Japan) system with a proximal clamp and cut-down technique above the occlusion level (Fig. 1). When the common carotid artery

Fig. 1 Schemas of direct carotid artery perfusion was transected, we removed all observable thrombi from the false lumen. DCP was introduced using the SCP circuit. After reaching the target body temperature, the aortic arch was opened, and an additional cerebral perfusion cannula was inserted to establish SCP $\left(10 \mathrm{ml} \times \mathrm{kg}^{-1} \times \mathrm{min}^{-1}\right.$, Fig. 1). The cerebral perfusion pressure was measured by the tip of each cerebral perfusion cannula, and continuous bilateral cerebral regional oxygen saturation was monitored with INVOS ${ }^{\text {TM }}$ Cerebral/Somatic Oximetry Adult Sensors throughout the surgery. A branch of the artificial graft was anastomosed to the common carotid artery with ligation of the proximal site (Fig. 1).

\section{Statistical procedures}

Continuous variables are expressed as mean $\pm \mathrm{SD}$ or median (range), and categorical variables are expressed as the number $(\%)$ of patients. Categorical variables were analyzed using Fisher's exact test. Continuous variables were compared using the Student's $t$ test, whereas nonparametric variables were analyzed using the Mann-Whitney $U$ test. All data analyses were performed with the JMP 14.1 software (SAS Institute, Cary, NC, USA). Statistical significance was defined as $p<0.05$.

\section{Results}

The patients' characteristics and preoperative data are summarized in Table 1. A total of 91 patients were admitted for AAAD involving the ascending aorta. Two patients who needed resuscitation owing to cardiopulmonary arrest because of coronary malperfusion and aortic rupture were excluded. Among the patients, 19 (21\%) presented
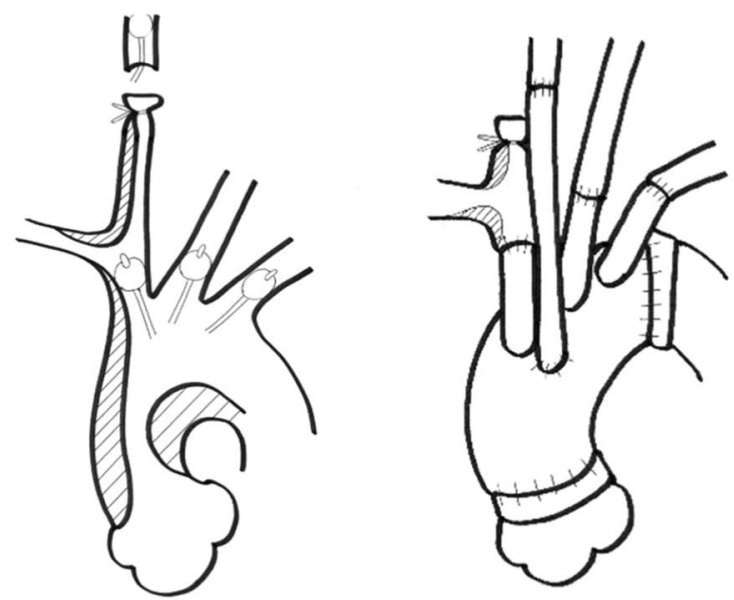

Direct carotid artery cannulation with other branch perfusion
Postoperative schema 
Table 1 Characteristics of patients

\begin{tabular}{|c|c|c|c|}
\hline & $\operatorname{SCP}(n=12)$ & $\mathrm{DCP}(n=7)$ & $\mathrm{p}$ \\
\hline Age (years) & $70(45-84)$ & $69(39-84)$ & 0.826 \\
\hline Sex (male, \%) & $10(83)$ & $7(100)$ & 0.160 \\
\hline Hypertension (\%) & $8(67)$ & $7(100)$ & 0.039 \\
\hline History of smoking (\%) & $8(67)$ & $6(86)$ & 0.348 \\
\hline $\begin{array}{l}\text { Chronic respiratory disease } \\
(\%)\end{array}$ & $1(8)$ & 0 & 0.329 \\
\hline Chronic kidney disease (\%) & $1(8)$ & $1(14)$ & 0.329 \\
\hline $\begin{array}{l}\text { Coronary artery disease } \\
(\%)\end{array}$ & $2(17)$ & 0 & 0.160 \\
\hline Neurological symptom (\%) & $4(30)$ & $4(57)$ & 0.311 \\
\hline $\begin{array}{l}\text { Other organ malperfusion } \\
(\%)\end{array}$ & $6(50)$ & $1(14)$ & 0.105 \\
\hline $\begin{array}{l}\text { Aortic valve regurgitation } \\
(\%) \text { (moderate to severe) }\end{array}$ & $3(25)$ & $2(28)$ & 0.865 \\
\hline Cardiac tamponade (\%) & $1(8)$ & $1(14)$ & 0.688 \\
\hline Shock hemodynamics (\%) & $1(8)$ & 0 & 0.329 \\
\hline $\begin{array}{l}\text { Cardiac arrest, resuscita- } \\
\text { tion }(\%)\end{array}$ & 0 & 0 & 0 \\
\hline GERAADA socre & $13.5(8.3-24.2)$ & $13.1(9.2-19.6)$ & 0.811 \\
\hline
\end{tabular}

$D C P$ direct carotid artery perfusion, GERAADA German Registry for Acute Aortic Dissection Type A, $S C P$ selective cerebral perfusion

Statistical significance was set at $p<0.05$

with involvement of the common carotid artery and were included in this study. The mean age was 69 years (range, 39-84 years); 17 patients were male (89\%). There were no patients with a family history of aortic dissection. Two (11\%) patients presented with hemodynamic instability owing to cardiac tamponade; however, their consciousness was intact. In 19 patients with involvement of the common carotid artery, the right common carotid artery was severely stenosed in 16 (84\%) patients and the left was stenosed in the remaining three (16\%). Twelve patients $(63 \%)$ underwent conventional SCP (Fig. 2a-c) and seven (37\%) underwent DCP (Fig. 3a-d). No significant differences were found in the preoperative characteristics except for medical history of hypertension between the conventional SCP and DCP groups. All patients in the DCP group had a medical history of hypertension compared with eight patients (67\%) in the SCP group $(p=0.039)$. There were no patients with a history of transient cerebrovascular disease; however, there was one case with parapledia owing to past spinal cord injury. Moreover, although there was no significant difference, six patients (50\%) in the SCP group developed other organ malperfusion $(p=0.105)$ : in the coronary artery in three cases, mesenteric artery in one, and peripheral artery in two. Conversely, one patient $(14 \%)$ in the DCP group developed peripheral artery malperfusion. The GERAADA score was 13.1 (range 8.3-24.2) in the SCP group and 13.5 (range 9.2-19.6) in the DCP group ( $p=0.811$ ).

Operative procedures and postoperative outcomes are summarized in Table 2. Time from onset to perfusion was 341 (207-501) minutes in SCP group and 427 (172-594) minutes in DCP group $(p=0.491)$. All patients were weaned from cardiopulmonary bypass uneventfully. Central aortic repair, hemiarch, and partial and total arch replacement were performed in 3, 5, and 11 patients each. No significant differences were found in terms of operative procedures and postoperative outcomes except for concomitant procedures, postoperative neurological worsening, and late mortality between the groups. SCP was associated with a significantly higher incidence of postoperative neurological worsening $(p=0.018)$ and late mortality $(p=0.039)$. Two patients in the conventional SCP group died of extensive cerebral infarction (Fig. 2c) during hospitalization. Two patients $(11 \%)$ in postoperative coma developed respiratory insufficiency, and percutaneous tracheostomy was required. Late mortality occurred in two (11\%) conventional SCP cases: one patient died of heart failure 3 months after surgery and
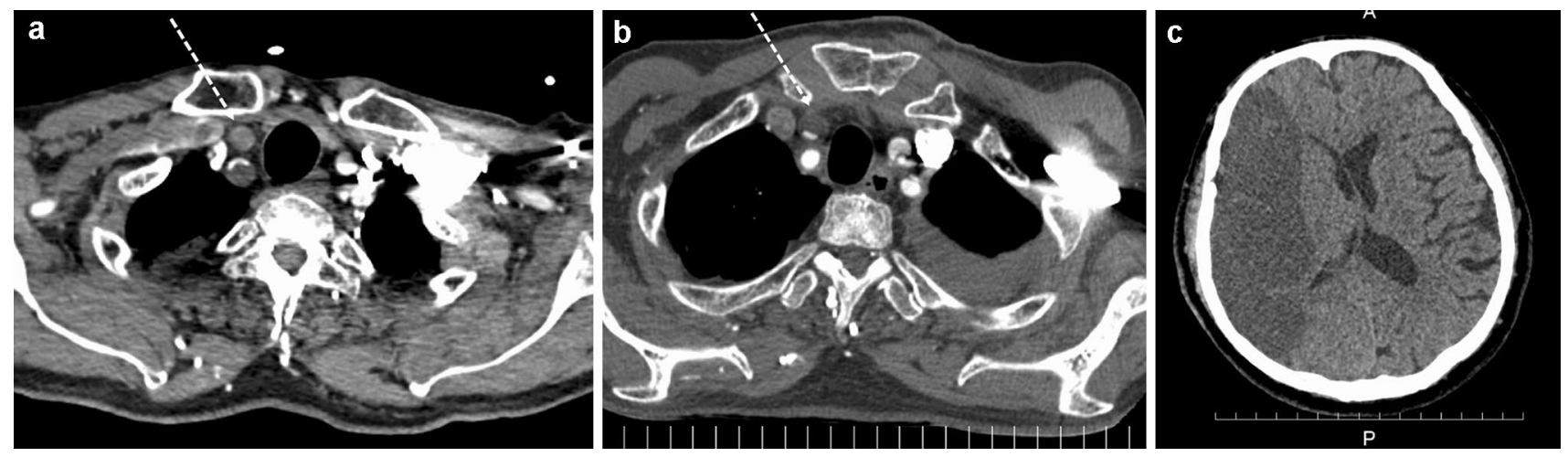

Fig. 2 a Preoperative contrast-enhanced computed tomography image of a patient in the selective cerebral perfusion group showing the right common carotid artery (white dotted arrow) with a severely narrowed true lumen. b Postoperative contrast-enhanced computed tomography image of the patient showing the remaining narrowed true lumen (white dotted arrow). c Postoperative head computed tomography image of the patient showing broad cerebral infarction 

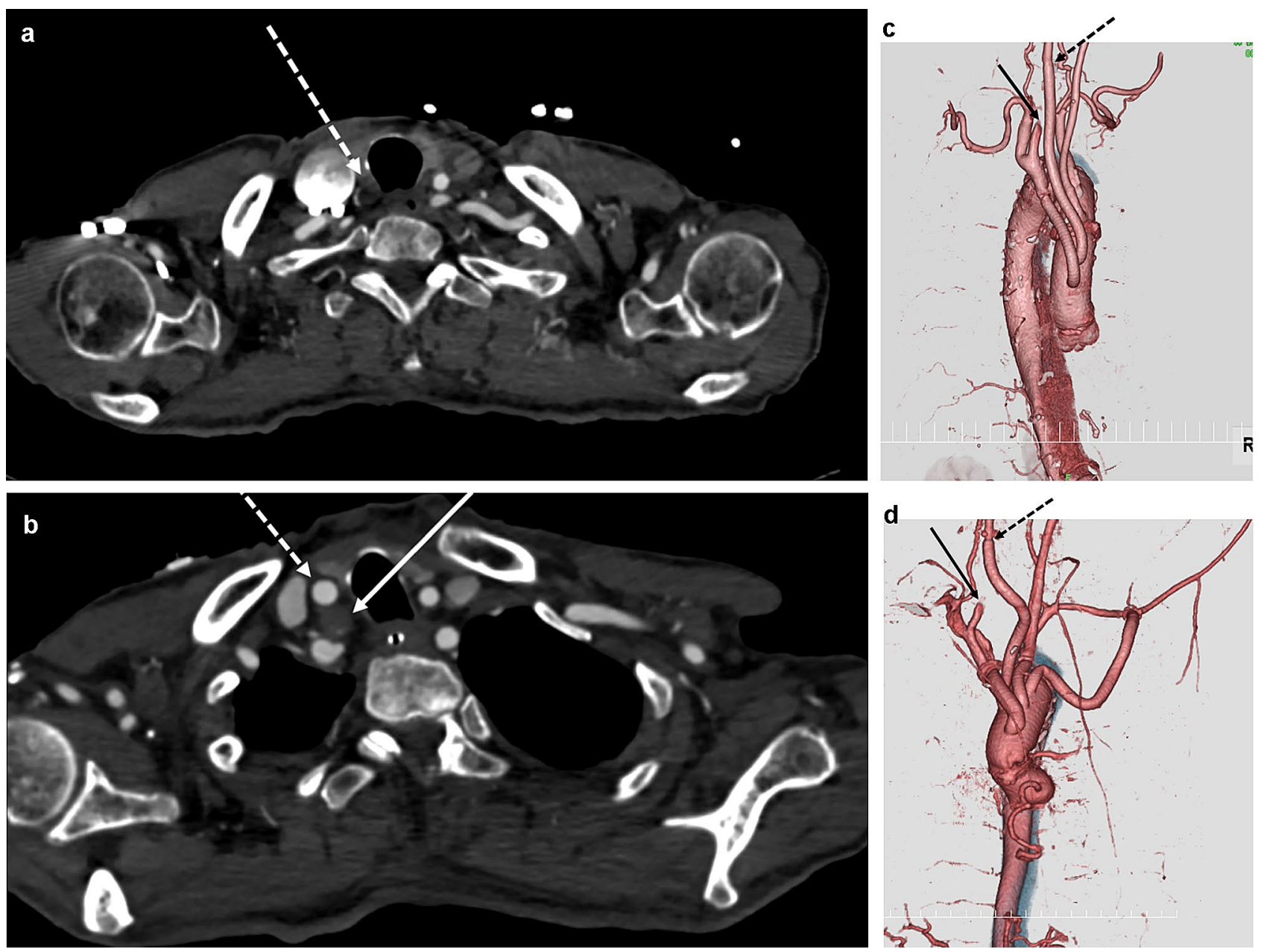

Fig. 3 a Preoperative contrast-enhanced computed tomography image of a patient in the direct carotid artery perfusion (DCP) group showing the right common carotid artery (white dotted arrow) with a severely narrowed true lumen. b Postoperative contrast-enhanced computed tomography image of the patient showing improvement

another patient died of unknown causes 15 months after surgery. During follow-up, a major aortic event occurred in one (5\%) DCP case, in which the patient needed additional aortic repair after 10 days owing to hemolysis associated with the internal felt strip.

The pre- and post-neurological changes are shown in Fig. 4a. On arrival, eight (42\%) patients had neurological symptoms: four in the SCP group and four in the DCP group. The remaining 11 (58\%) patients were neurologically asymptomatic: eight in the SCP group and three in the DCP group. Among 12 patients in the conventional SCP group, five developed worsening of neurological symptoms after open surgery (Fig. 2c). Only one patient with hemiparesis improved to become asymptomatic postoperatively. The remaining six patients presented no significant changes in neurological outcomes. Among seven patients in the DCP group, none experienced worsening of neurological of compression (white dotted arrow) and ligated proximal site (white arrow) in the right common carotid artery. c, d Postoperative threedimensional computed tomography images of patients in the DCP group showing no abnormality in the right carotid artery anastomosis (black dotted arrow) and the ligated proximal site (black arrow)

symptoms, although five showed no significant changes in neurological symptoms. Neurological symptoms improved postoperatively in two patients with hemiparesis.

Postoperative contrast-enhanced CT was performed in 16 patients (Fig. 4b). In the conventional SCP group, three patients showed improvement of stenosis in the carotid artery, three showed moderate residual stenosis, and the remaining three showed severe residual stenosis. In the DCP group, six patients improved and only one showed moderate residual stenosis. There was no significant difference between the groups $(p=0.050)$; however, there was a trend that patients in the DCP group showed improvement of stenosis in the carotid artery.

We assessed the change in values of continuous bilateral cerebral regional oxygen saturation from the the start of cerebral perfusion, $10 \mathrm{~min}$ after, $30 \mathrm{~min}$ after, $60 \mathrm{~min}$ after the start of cerebral perfusion and post surgery. There were no 
Table 2 Operative procedures and outcomes

\begin{tabular}{llll}
\hline & SCP $(n=12)$ & DCP $(n=7)$ & $p$ \\
\hline Central aortic repair (HAR, PAR, TAR) & $3: 2: 7$ & $0: 3: 4$ & \\
Concomitant procedure & CABG 3, AVR 1 & 0 & 0.039 \\
Onset to perfusion time (minutes) & $341(207-501)$ & $427(172-594)$ & 0.491 \\
Operation time (minutes) & $489(302-650)$ & $482(394-580)$ & 0.958 \\
CPB (minutes) & $275(162-378)$ & $254(185-355)$ & 0.776 \\
ACC (minutes) & $133(104-199)$ & $152(100-236)$ & 0.600 \\
SCP (minutes) & $166(32-212)$ & $166(84-205)$ & 0.749 \\
CA (minutes) & $58(33-77)$ & $49(38-65)$ & 0.320 \\
Body temperature $\left({ }^{\circ} \mathrm{C}\right)$ & $23.8(21.1-24.7)$ & $23.5(21.8-24.5)$ & 0.873 \\
ICU stay (days) & $4(2-25)$ & $5(3-21)$ & 0.885 \\
Hospitalization (days) & $26(7-69)$ & $34(18-49)$ & 0.653 \\
Neurological outcomes $(\%)$ & $5(42)$ & $4(57)$ & 0.049 \\
Neurological worsening $(\%)$ & $5(42)$ & 0 & 0.018 \\
Respiratory complication $(\%)$ & $1(8)$ & $1(14)$ & 0.688 \\
Renal complication $(\%)$ & 0 & 0 & \\
Early mortality (\%) & $2(17)$ & 0 & 0.160 \\
Late mortality (\%) & $2(17)$ & 0 & 0.039 \\
Aortic events $(\%)$ & 0 & $1(14)$ & 0.348 \\
\hline
\end{tabular}

Statistical significance was set at $p<0.05$

$A C C$ aortic cross clamp, $A V R$ aortic valve replacement, $C A$ circulatory arrest, $C A B G$ coronary artery bypass grafting, $C P B$ cardiopulmonary bypass, $D C P$ direct carotid artery perfusion, HAR hemiarch replacement, $I C U$ intensive care unit, $P A R$ partial arch replacement, $S C P$ selective cerebral perfusion, TAR total arch replacement significant changes in the values between the SCP and DCP groups (Fig. 4c, $p=0.551$, 0.926. 0.601 and 0.771). There were no significant changes in the values in the presence or absence of worsening of postoperative neurological symptoms (Fig. 4d, $p=0.425,0.801,0.444$ and 0.601).

\section{Discussion}

This study showed that DCP during AAAD repair resulted in good postoperative neurological outcomes and late mortality compared with conventional SCP. In particular, in patients for whom DCP was applied, no worsening of any neurological condition recorded at admission was present postoperatively, although there was no improvement in postoperative neurological outcomes when cerebral infarction had already developed on arrival.

The pathophysiology of brain complications in acutely dissected patients is often multifactorial and frequently coexists with circulatory collapse, sequelae of acute hypoxia, and thromboembolism originating from the false lumen [7]. Benninger et al. showed that thromboembolism is an essential mechanism in isolated carotid artery dissection [8]. Furukawa et al. reported that patients with partial or complete thrombosis of the arch vessels are at a high risk of cerebral malperfusion, especially when the false lumen of the innominate artery is occluded [9]. Gomibuchi et al. stated that postoperative neurological deficiency is caused not only by disturbed blood flow in an occluded or stenotic carotid artery but also by a thromboembolism owing to thrombi derived from a thrombosed false lumen [6]. Gaul et al. reported that in one-third of all patients with preoperative stroke, ischemic stroke was not caused by an extension of the dissection toward the supra-aortic vessels. Instead, it was the result of other etiological mechanisms such as thromboembolism or severe hypotension [10]. In our institute, in some cases, a thrombus in the true lumen from the false lumen of the common carotid artery is detected intraoperatively. Therefore, we always remove thrombi from the false lumen before the initiation of SCP. Once SCP is established, thrombi in the false lumen may enter the true lumen through a small entry. If re-entry occurrs at the distal site and we anastomose at the orifice of the brachiocephalic artery, it is possibile that the thrombi are transferred from the false lumen via the distal re-entry site [6].

"Imaging cerebral malperfusion" introduced by Gomibuchi et al. is a crucial concept for detecting brain ischemia regardless of symptoms in the preoperative difficult situation [6]. Patients with a subclinical imaging cerebral malperfusion preoperatively maintain cerebral circulation by collateral circulation via the circle of Willis, and it is therefore important not to disrupt the cerebral circulation. Moreover, it does not matter whether there are preoperative neurological symptoms because it is unclear which cases will develop 


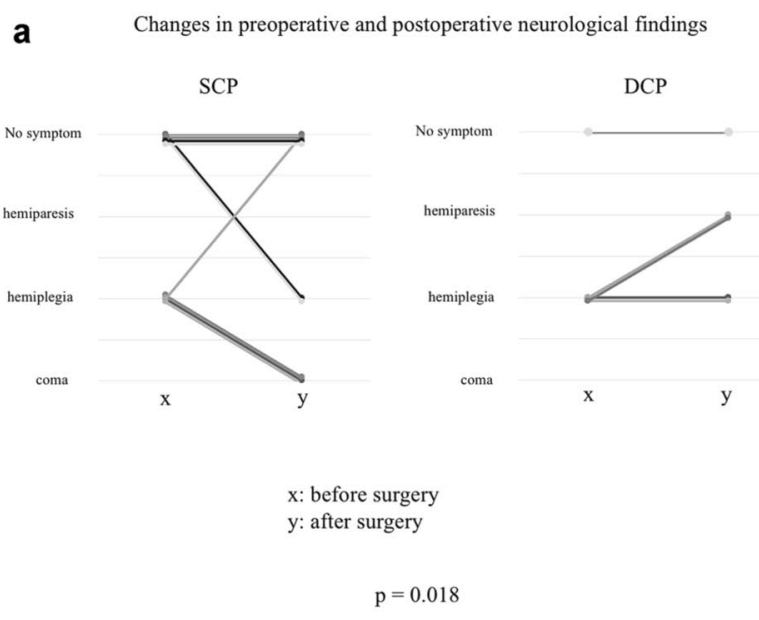

b Changes in carotid artery stenosis on preoperative and postoperative CT

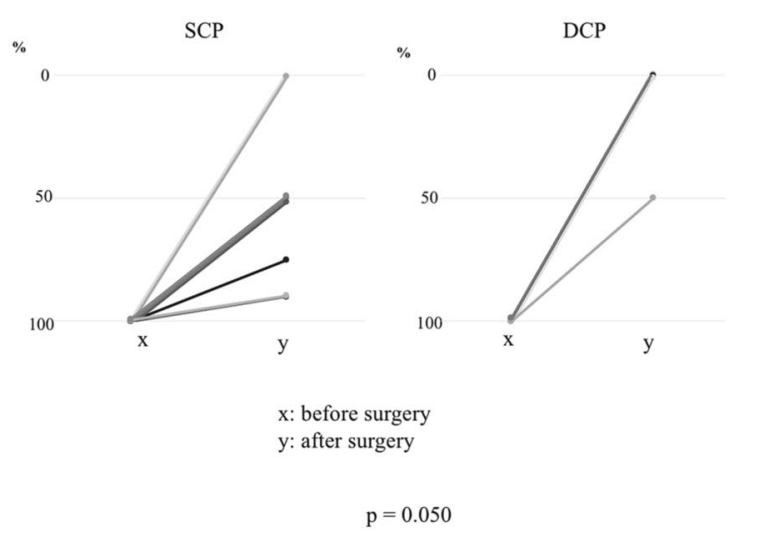

Fig. 4 a Changes in preoperative and postoperative neurological findings. b Changes in carotid artery stenosis on preoperative and postoperative CT. c Changes in cerebral regional oxygen saturation from

neurological sequelae after surgery. Because it is unclear whether cerebral infarction will develop, sufficient time is not available to examine brain perfusion using multimodalities such as head magnetic resonance imaging and enhanced head CT; moreover, the carotid artery can be easily exposed. Based on the results of this study, we decided to use the DCP for all cases.

Immediate surgery for patients with cerebral malperfusion can lead to acceptable neurologic outcomes; however, most preoperative neurologic symptoms are irreversible [3, 4]. Restoration of cerebral blood flow via conventional central aortic repair with SCP may be very difficult if severe stenosis or occlusion of the common carotid arteries is present. We experienced some cases that developed irreversible neurological deficiency with conventional SCP. There have been various strategies for brain protection in AAAD patients with brain malperfusion. Munakata et al. reported the performance of "controlled reperfusion [19]," in which
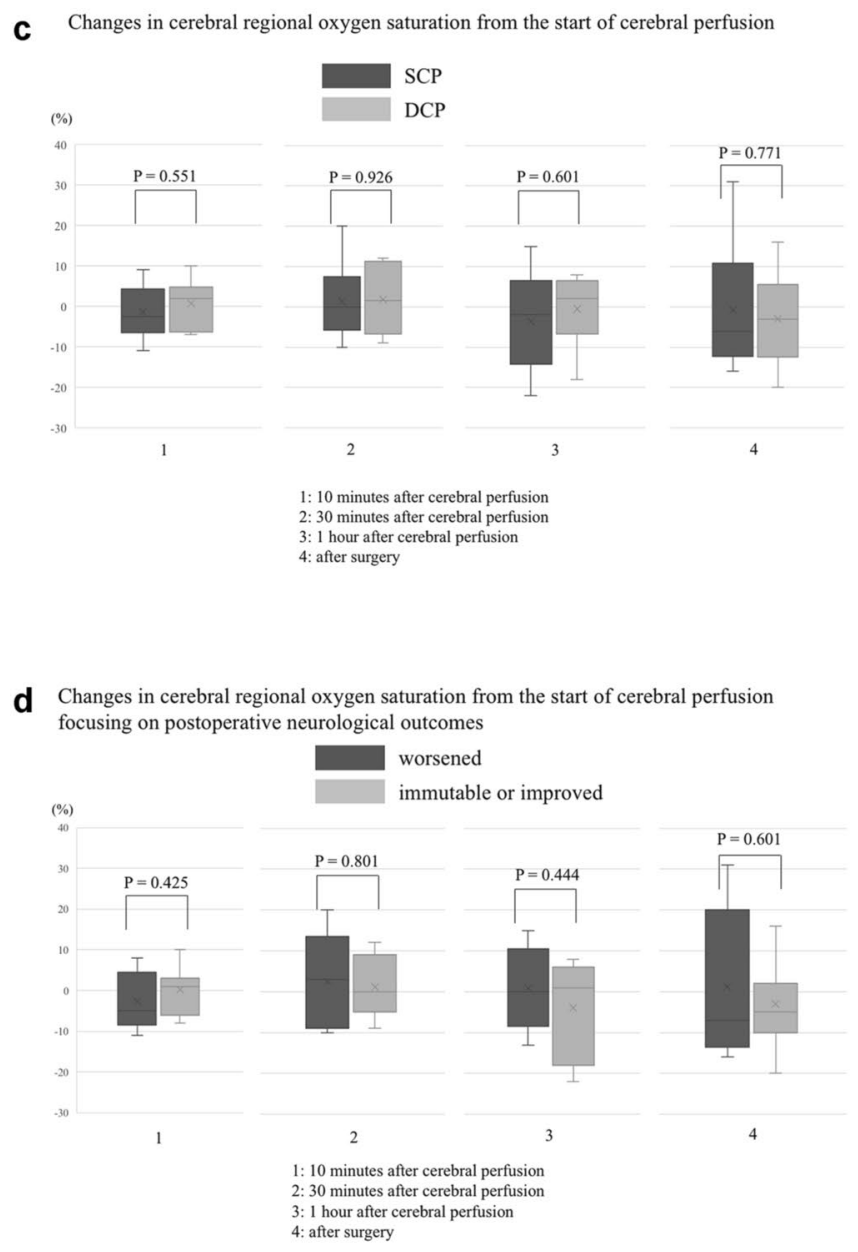

the start of cerebral perfusion. $\mathbf{d}$ Changes in cerebral regional oxygen saturation from the start of cerebral perfusion focusing on postoperative neurological outcomes

perfusion with cold blood was used first to reduce reperfusion injury after prolonged ischemia. Igarashi et al. removed a thrombus in the false lumen of the common carotid artery to restore the true lumen in two cases [20]. Carotid stenting may be a good alternative to produce better results in this subset [21]. However, catheter manipulations have the potential to injure the thin aortic wall or mobilize the thrombus in the false lumen, leading to cerebral embolism. Ueyama et al. reported a case where the right common carotid artery was first bypassed, followed by aortic repair [22]. Regarding the procedure of arterial cannulation, direct brachiocephalic artery cannulation is a less time-consuming and reliable method to restore cerebral perfusion [23]; however, there is the concern of turbulent blood flow with that method [24]. Matsushita et al. reported 52 cases of transapical cannulation, and they converted to another perfusion route to resolve intraoperative malperfusion syndrome in five cases (9.6\%) [25]. Furthermore, a 5-11\% incidence of cerebral 
complications has been reported using central cannulation [14]. Samely, direct ascending aortic cannulation was established as a promising new method; however, it is suboptimal for brain protection [14]. Axillary artery perfusion may not restore the true lumen when there is a compressed true lumen at the distal site of the carotid artery and may cause systemic hypoperfusion [4]. In the present study, in conventional SCP with axillary artery perfusion, neurological symptoms worsended in five (42\%) patients after open surgery. Axillary artery perfusion may not sufficiently perfuse to the right carotid artery because of severe stenosis associated with the compression of the true lumen, even if continuous bilateral regional oxygen saturation is maintained throughout the surgery.

Direct carotid artery perfusion has been introduced to resolve preoperative cerebral malperfusion $[7,15,16]$. Luehr et al. reported that extra-anatomic aorto-carotid bypass was performed in 23 patients who had cerebral malperfusion owing to the occlusion of the carotid artery secondary to aortic dissection [26]. Okita et al. reported a simple bypass circuit between the common femoral artery and right common carotid artery performed in the emergency room [7]. Surgical access to the common carotid artery is very easy and quick and is therefore suitable for emergencies. However, it is sometimes difficult to establish this perfusion system in the emergency room. Moreover, there are some differences between our approach and their common carotid artery perfusion. They introduced this perfusion system in the emergency room only for patients with neurological symptoms. Furthermore, they used the femoral artery as inflow using a double-lumen or Y-shaped line to gain prompt brain perfusion before aortic repair. In the present study, direct perfusion to the carotid artery was introduced in the operating room using the SCP circuit. Although their main concept is based on achieving prompt perfusion in the ischemic carotid artery, our goal is to obtain reliable cerebral perfusion and prevent thromboembolism at the distal site during surgery. Sasaki et al. considered that definitive blood flow restoration under direct vision with hypothermic circulatory arrest is of utmost importance to prevent creating a new intimal tear and embolism [15]. Among their nine cases, three developed new cerebral infarction, although two of them did not have any neurological symptoms at admission. The DCP method improves postoperative results by preventing this thromboembolism at revascularization in addition to the reversible brain damage.

In the present study, there were no significant changes between the groups with respect to continuous bilateral cerebral regional oxygen saturation values. Moreover, there were no significant difference in values due to postoperative neurological outcomes. Cerebral regional oxygen saturation monitored with INVOS is reliable for detecting changes in blood flow [27]; however, it cannot detect fine changes during continuoous perfusion with cardiopulmonary bypass [28]. The reason why continuous bilateral cerebral regional oxygen saturation is maintained in the presennt study although cerebral infarction occurs, may be the existence of collateral circulation perfused by cardiopulmonary bypass.

The GERAADA score is a simple effective tool to predict the 30-day mortality rate for patients undergoing surgery for AAAD [17, 18]. Malperfusion of any organ system, particularly multiple organ ischemia, has been shown to be a strong independent predictor of the 30-day mortality rate. In the present study, there was no significant difference between the goups with respect to the GERAADA score. Moreover, the value of the GERAADA score in the present study was not higher than that in the other cohort without the involvement of the carotid artery [18]. Although the GERAADA socre may be identified as an independent predictor of the 30-day mortality rate, it might not be related to neurological outcomes.

There are unsolved problems with this method. Moreover, the management strategy for patients with preoperative coma remains unknown. According to Tanaka et al., patients in preoperative coma for more than $3 \mathrm{~h}$ after onset should be excluded as candidates for immediate central aortic repair surgery [29]. In this study, time from onset to perfusion were 341 in the SCP group and 427 min in the DCP group; which could be shortend. Preoperative focal neurologic symptoms were reversible in some cases, and postoperative rehabilitation could achieve acceptable recovery. However, it is difficult to determine which patients with neurological symptoms will recover after surgery [3, 4]. Although five patients in the DCP group had hemiparesis at discharge, their symptoms gradually improved during follow-up. Furthermore, continuous bilateral cerebral regional oxygen saturation is a reliable monitoring method for detecting changes in intracranial blood flow of patients; however, it cannot constitute absolute evaluation for brain protection [28]. Therefore, the optimal indications and contraindications for brain malperfusion remain unclear. Further studies regarding these problems are warranted.

\section{Study limitations}

This study had some limitations. First, relatively few patients were included owing to the rarity of this condition. Second, this was a retrospective single-center experience lacking any form of randomization. Third, the surgical technique for AAAD has evolved during the time of this study. To resolve these limitations, a multi-institutional study is needed. Further studies on brain mechanisms at the time of reperfusion during surgery are warranted. Moreover, public-health management strategies that would reduce the time from onset to admission are needed. 


\section{Conclusion}

DCP in AAAD with cerebral malperfusion was acceptable. Although this method did not improve neurological symptoms that had already developed on arrival, it prevented worsening of neurological symptoms. Furthermore, it may prevent the onset of new infarction after cerebral reperfusion during surgery in asymptomatic cases.

Acknowledgements We thank the Honyaku Center for reviewing and editing the manuscript. We also thank our colleagues for their helpful comments.

\section{Declarations}

Conflict of interest Kayo Sugiyama, Hirotaka Watanuki, Yasuhiro Futamura, Masaho Okada, Tatsunori Oiwa, Satoshi Makino, and Katsuhiko Matsuyama have no conflict of interest. We did not receive any financial support for this study.

\section{References}

1. Bonser RS, Ranasinghe AM, Loubani M, Evans JD, Thalji NMA, Bachet JE, et al. Evidence, lack of evidence, controversy, and debate in the provision and performance of the surgery of acute type A aortic dissection. JACC. 2011;58:2455-74.

2. Czerny M, Schoenhoff F, Etz C, Englberger L, Khaladj N, Zierer A, et al. The impact of preoperative malperfusion on outcome in acute type A aortic dissection. JACC. 2015;65:2628-35.

3. Gaul C, Dietrich W, Erbguth FJ. Neurological symptoms in aortic dissection: a challenge for neurologists. Cerebrovasc Dis. 2008;26:1-8.

4. Orihashi K. Cerebral malperfusion in acute aortic dissection. Surg Today. 2016;46:1353-61.

5. Gomibuchi T, Seto T, Komatsu M, Tanaka H, Ichimura H, Yamamoto T, et al. Impact of frailty on outcomes in acute type A aortic dissection. Ann Thorac Surg. 2018;106:1349-55.

6. Gomibuchi T, Seto T, Naito K, Chino S, Mikoshiba T, Komatsu M et al. Strategies to improve outcomes for acute type A aortic dissection with cerebral malperfusion. Eur J Cardio-Thorac Surg. 2020; $0 ; 1-8$.

7. Okita Y, Ikeno Y, Yokawa K, Koda Y, Henmi S, Gotake Y, et al. Direct perfusion of the carotid artery in patients with brain malperfusion secondary to acute aortic dissection. Gen Thorac Cardiovasc Surg. 2019;67:161-8.

8. Benninger DH, Georgiadis D, Kremer C, Studer A, Nedeltchev K, Baumgartner RW. Mechanism of ischemic infarct in spontaneous carotid dissection. Stroke. 2004;35:482-5.

9. Furukawa T, Uchida N, Takahashi S, Yamane Y, Mochizuki S, Yamada K, et al. Management of cerebral malperfusion in surgical repair of acute type A aortic dissection. Eur J Cardiothorac Surg. 2017;52:327-32.

10. Gaul C, Dietrich W, Friedrich I, Sirch J, Erbguth FJ. Neurological symptoms in type A aortic dissections. Stroke. 2007;38:292-7.

11. Rylski B, Urbanski PP, Siepe M, Beyersdorf F, Bachet J, Gleason TG, et al. Operative techniques in patients with type A dissection complicated by cerebral malperfusion. Eur J Cardio-Thorac Surg. 2014;46:156-66.

12. Orihashi K, Sueda T, Okada K, Takahashi S. Compressed true lumen in the innominate artery: a pitfall of right axillary arterial perfusion in acute aortic dissection. J Thorac Cardiovasc Surg. 2009;137:242-3.
13. Imanaka K, Kawata M, Matsuoka T, Yamabi H. Uncertainty of axillary artery perfusion during surgery for acute aortic dissection. Ann Thorac Surg. 2014;97:1781-2.

14. Conzelmann LO, Kayhan N, Mehlhorn U, Weigang E, Dahm M, Vahl CF. Reevaluation of direct true lumen cannulation in surgery for acute type A aortic dissection. Ann Thorac Surg. 2009;87:1182-6.

15. Sasaki H, Harada T, Ishitoya H, Sasaki O. Aorto-carotid bypass for type A acute aortic dissection complicated with carotid artery occlusion: no touch until circulatory arrest. Interact Cardiovasc Thorac Surg. 2020;31:263-5.

16. Urbanski PP, Irimie V, Wagner M. Cannulation and perfusion strategy in acute aortic dissection involving both common carotid arteries. Interact Cardiovasc Thorac Surg. 2015;21:557-9.

17. Czerny M, Siepe M, Beyersdorf F, Feisst M, Gabel M, Pilz M, et al. Prediction of mortality rate in acute type A dissection: the German Registry for Acute Type A Aortic Dissection score. Eur J Cardiothorac Surg. 2020;58:700-6.

18. Luher M, Merkle-Storms J, Gerfer S, Li Y, Krasivskyi I, Vehrenberg $\mathrm{J}$ et al. Evaluation of the GERAADA score for prediction of 30-day mortality in patients with acute type A aortic dissection. Eur J Cardio-Thorac Surg. 2020; 0: 1-6.

19. Munakata H, Okada K, Kano H, Izumi S, Hino Y, Matsumori M, et al. Controlled earlier reperfusion for brain ischemia caused by acute type A aortic dissection. Ann Thorac Surg. 2009;87:e27-8.

20. Igarashi $\mathrm{T}$, Takahashi $\mathrm{S}$, Takase $\mathrm{S}$, Yokoyama H. Intraoperative thrombectomy for occluded carotid arteries in patients with acute aortic dissection: report of two cases. Surg Today. 2014;44:1177-9.

21. Roseborough GS, Murphy KP, Barker PB, Sussman M. Correction of symptomatic cerebral malperfusion due to acute type I aortic dissection by transcarotid stenting of the innominate and carotid arteries. J Vasc Surg. 2006;44:1091-6.

22. Ueyama K, Otaki K, Koyama M, Kamiyama H. Urgent simultaneous revascularization of the carotid artery and ascending aortic replacement for type A acute aortic dissection with cerebral malperfusion. Gen Thorac Cardiovasc Surg. 2007;55:284-6.

23. Sakaguchi G, Komiya T, Tamura N, Obata S, Masuyama S, Kimura C, et al. Cerebral malperfusion in acute type A dissection: direct innominate artery cannulation. J Thorac Cardiothorac Surg. 2005;129:1190-1.

24. Shiiya N, Kunihara T, Kamikubo Y, Yasuda K. Isolation technique for stroke prevention in patients with a mobile atheroma. Ann Thorac Surg. 2001;72:1401-2.

25. Matsushita A, Manabe S, Tabata M, Fukui T, Shimokawa T, Takanashi S. Efficacy and pitfalls of transapical cannulation for the repair of acute type A aortic dissection. Ann Thorac Surg. 2012;93:1905-9.

26. Luehr M, Etz CD, Nozdryxkowski M, Lehmkuhl L, Misfeld M, Bakhtiary $\mathrm{F}$ et al. Extra-anatomic revascularization for preoperative cerebral malperfusion due to distal carotid artery occlusion in acute type A aortic dissection. Eur JCardio-Thorac Surg. 2015; 1-8.

27. Olsson C, Thelin S. Regional cerebral saturation monitoring with near-infrared spectroscopy during selective antegrade cerebral perfusion: diagnostic performance and relationship to postoperative stroke. J Thorac Cardiovasc Surg. 2006;131:371-9.

28. Davie SN, Grocott HP. Impact of extracranial contamination on regional cerebral oxygen saturation. A comparison of three cerebral oximetry technologies. Anesthesiology. 2012;116:834-40.

29. Tanaka H, Okada K, Yamashita T, Morimoto Y, Kawanishi Y, Okita Y. Surgical results of acute aortic dissection complicated with cerebral malperfusion. Ann Thorac Surg. 2005;80:72-6.

Publisher's Note Springer Nature remains neutral with regard to jurisdictional claims in published maps and institutional affiliations. 